EGU21-15968

https://doi.org/10.5194/egusphere-egu21-15968

EGU General Assembly 2021

(c) Author(s) 2021. This work is distributed under

the Creative Commons Attribution 4.0 License.

\title{
Thermal fractionation of soil organic matter produces fine-scale distributions of SOM age
}

\author{
Shane Stoner ${ }^{1,2}$, Carlos Sierra ${ }^{1}$, Marion Schrumpf ${ }^{1}$, Sebastian Dötterl ${ }^{2}$, and Susan Trumbore ${ }^{1}$ \\ ${ }^{1}$ Max Planck Institute for Biogeochemistry, Biogeochemical Processes, Jena, Germany (sstoner@bgc-jena.mpg.de) \\ ${ }^{2}$ ETH-Zürich, Department of Environmental Systems Science, Zürich, Switzerland
}

Soil organic matter (SOM) is a complex collection of organic molecules of varying origin, structure, chemical activity, and mineral association. A wide array of laboratory methods exists to separate SOM based on qualitative, biological, chemical, and physical characteristics. However, all present conceptual and logistical limitations, including the requirement of a substantial amount soil material.

An newly applied alternative method of fractionation relies on a conceptual analogue between biochemical stability in soil and thermal stability, e.g. more persistent SOM will require higher temperatures (greater energy inputs) to decompose than less persistent SOM. This accounts for both chemical complexity and mineral association as main factors in determining SOM persistence.

In this method, carbon is released by heating SOM to $900^{\circ} \mathrm{C}$ at a constant rate. The peaks of carbon release are grouped into activation energy pools, $\mathrm{CO}_{2}$ is collected, and analyzed for ${ }^{13} \mathrm{C}$ and ${ }^{14} \mathrm{C}$. We seek to describe in finer detail the distribution of soil radiocarbon by adding another fractionation step following a different paradigm of SOM stability, and explore mineralogical effects on SOM quality and stability using thermal analysis, radiocarbon, and gas chromatography.

Here, we analyzed bulk soil and soil fractions derived from density separation and chemical oxidation, as well as mineral horizons dominated by diverse mineralogies. Density fractions contained a wide range of radiocarbon activities and that young SOM is stabilized across multiple fractions, likely due to organomineral complexation. Initial results showed that soil minerals with limited stabilization potential released $C$ at lower temperatures than those with diverse stabilization mechanisms. High-temperature sub-fractions contained the oldest carbon across fractions and minerals, thus supporting the assumption that thermal stability can be used as a limited analogue for stability in soil. We present a fine-scale distribution of radiocarbon in SOM and discuss the potential of this method for comparison with other fractionation techniques. 